\title{
The "Tipping Point" for Breast Cancer Mortality Decline Has Resulted from Size Reductions Due to Mammographic Screening
}

\author{
Blake Cady, $\mathrm{MD}^{1,2}$, James S. Michaelson, $\mathrm{PhD}^{3}$, and Maureen $\mathrm{A}$. Chung, $\mathrm{MD}, \mathrm{PhD}^{4}$ \\ ${ }^{1}$ Department of Surgery, Cambridge Breast Center, Cambridge Hospital, Cambridge, MA; ${ }^{2}$ Harvard Medical School, \\ Boston, MA; ${ }^{3}$ Division of Surgical Oncology, Massachusetts General Hospital, Harvard Medical School, Boston, MA; \\ ${ }^{4}$ Department of Surgery, Rhode Island Hospital, Brown University School of Medicine, Providence, RI
}

Considering the declining breast cancer mortality in the United States, it is apparent that a "tipping point" was reached between 1986 and 1991, most apparent after 1990. For the first time since 1930, the previously unyielding ageadjusted breast cancer mortality rate began a continuous decline, starting about 5 years after the upsurge in use of mammographic screening in the 1980s (Fig. 1). ${ }^{1}$ This mortality decline had reached $30 \%$ in the entire United States by 2007 (Fig. 2; http://www.seer.cancer.gov) and had reached as much as $48 \%$ in Delaware, $41 \%$ in Rhode Island, and $40 \%$ in Massachusetts (http://statecancerprofiles.cancer.gov), the three highest states in mammography use recorded by the 2006 Behavioral Risk Factor Surveillance System (BRFSS) telephone surveys (http://cdc.gov/brfss/). The BRFSS estimates mammography use at least every 2 years by women over the age of 40 . In contrast, mortality decline has been only $11 \%$ in Utah, $12 \%$ in Mississippi, and $14 \%$ in Oklahoma, three of the four lowest states in BRFSS reports of screening incidence (Fig. 3; http://statecancerprofiles. cancer.gov). Although the BRFSS rates may be somewhat exaggerated because they are based on women's recall, the resultant relative ranking is undoubtedly correct because survey techniques are similar across the country. States with the greatest decline in mortality are also states with high initial incidence and mortality from breast cancer due to well-established demographic features such as older age, higher socioeconomic status, and higher education level. ${ }^{2}$

(C) Society of Surgical Oncology 2011

Published Online: 26 January 2011

B. Cady, MD

e-mail: bcady123@comcast.net
Because mammographic screening reduces mortality by earlier detection of a cancer with a biologically progressive disease course in most situations, a reduced rate of advanced breast cancers in a population is a surrogate for the success of screening. Here, we analyze the role of the steady change in tumor size as a result of breast cancer screening and attempt to demonstrate a temporal and possible causal relationship between changes in tumor size and the beginning of the decrease in breast cancer mortality (that is, the tipping point). The mean maximum diameter of all breast cancers was about $3.5 \mathrm{~cm}$ before 1950, about $3.0 \mathrm{~cm}$ in 1968 (just before the introduction of mammography), reached about $2.5 \mathrm{~cm}$ by 1987 , and had a progressive further decline to less than $2.0 \mathrm{~cm}$ by $2006 .^{3-5}$ The median maximum diameter of breast cancer, a better description of the usual presentation because it eliminates the bias of the extended "tail" of very large cancers, was $2.5 \mathrm{~cm}$ in $1968,2.0 \mathrm{~cm}$ by 1987 , and $1.5 \mathrm{~cm}$ after $2002 .^{3-5}$ Accompanying this overall size decrease, the axillary lymph node metastatic rate, which was over $60 \%$ in the 1950 s, usually with multiple node metastases, decreased to about $50 \%$ by 1968 , and thereafter progressively declined to less than $30 \%$ by $2006 .^{3-5}$ Equally important was the progressive decline in the number of lymph node metastases involved in women with invasive breast cancer through the years, so that now only one node metastasis is found in about $50 \%$ of patients who have regional nodal metastases, and all nodal metastases are limited to the excised sentinel nodes in up to two-thirds of patients. ${ }^{6,7}$

With the adoption of limited axillary node sampling by sentinel node biopsy, smaller volumes of nodal involvement have been recorded as pathologists more rigorously section and examine the few sentinel nodes submitted (median \# 2), which in turn technically increases the rate of nodal metastases by up to $20 \%$, a good example of the 


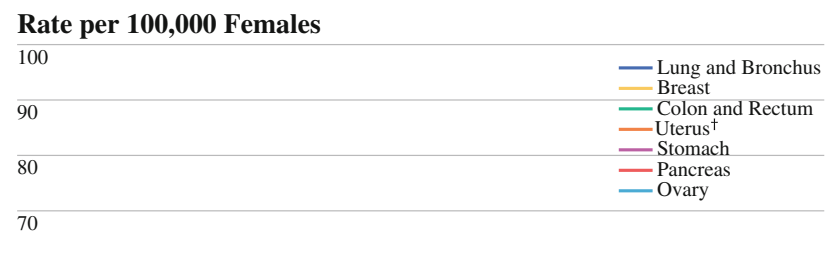

60
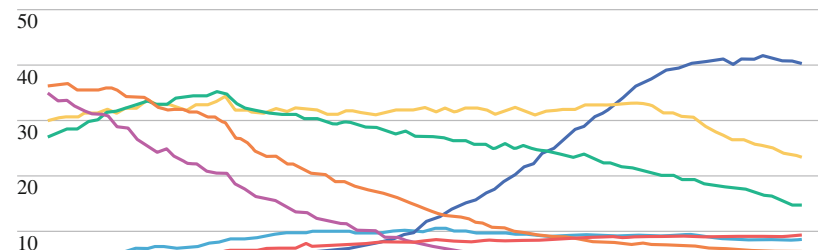
10

1930193519401945195019551960196519701975198019851990199520002005 Year of Death

FIG. 1 Annual age-adjusted cancer death rates among women for selected cancers, United States, 1930-2006. There was essentially no change in age adjusted mortality observed between 1930 and 1990, after which a continuing decline in age adjusted mortality has occurred through 2006 (Reprinted from Jemal A, Siegel R, Xu J, Ward E, Cancer statistics, 2010. CA: A Cancer Journal for Clinicians, n/a. doi:10.3322/caac.20073)

"Will Rogers" stage shifting phenomena. ${ }^{8}$ Compared to earlier axillary dissections where each of the many nodes submitted (median \# 16) were only pathologically sampled by a single section of each node, sentinel node analysis by pathologists is more sophisticated, and results in more frequent, but minor, nodal metastases, frequently only cell clusters or micrometastases $(<2 \mathrm{~mm}$ in size).

The third major morphological prognostic factor for mortality is tumor grade, more often low, or well differentiated, in small, mammographically discovered cancers, and high, or undifferentiated, in larger palpable breast cancers. ${ }^{9}$ The proportion of high-grade, poorly differentiated cancers has decreased in heavily screened populations, and the proportion of low-grade cancers has increased secondarily through the decrease in size resulting from detection earlier in their biological evolution., 5

The tipping point size, with its accompanying decreased proportion of lymph node metastases and high-grade cancers that has led to the onset of mortality decline, can be estimated at a mean maximum diameter of about $2.5 \mathrm{~cm}$ and a median maximum diameter of about $2.0 \mathrm{~cm}$, which was achieved after 1987. Thus, temporally, the tipping point size is related to the onset of the progressive mortality decline beginning about 1990, about 5 years after the large increase in mammographic screening of women that occurred in the 1980s. ${ }^{10}$

Previous decreases in size (and node metastatic rate) before mammographic screening, from $3.5 \mathrm{~cm}$ between 1929 and 1948 , to $3.0 \mathrm{~cm}$ by 1968 , occurred without

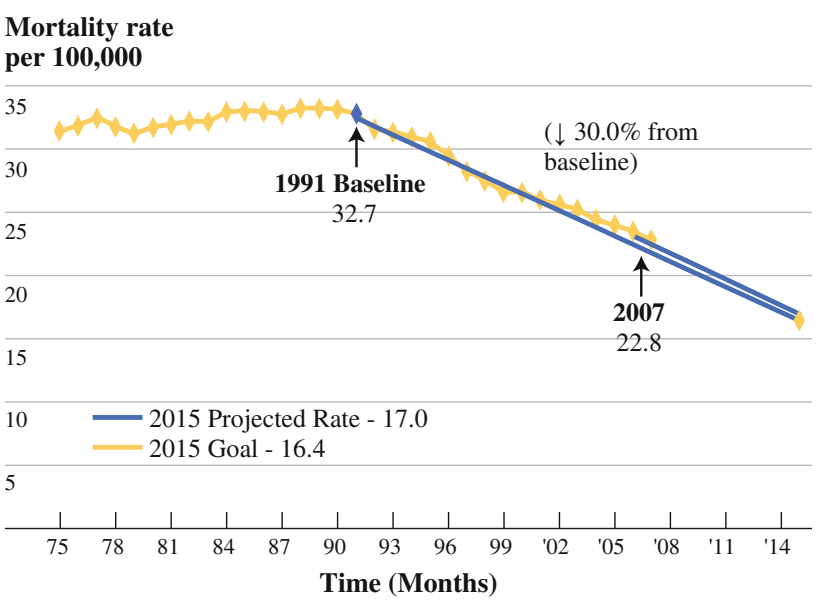

FIG. 2 Female breast cancer mortality rates from 1975 and projected rate to 2015. In 2007, mortality from breast cancer was down $30.3 \%$ from the 1991 baseline attributed to better ways to detect and treat breast cancer. The current trend to 2015 shows a $47.9 \%$ decrease from baseline. The latest joinpoint trend (1990-2007) shows a -2.2 annual percentage change (APC) in age-adjusted rates. Until over $90 \%$ of American women over age 40 are getting annual mammograms, the potential remains unrealized for a more substantial reduction in breast cancer mortality. Mortality rates are reported per 100,000 and are ageadjusted to 2000 U.S. standard population. (Reprinted from American Cancer Society analysis of female breast cancer (invasive) incidence rates: 2015 goal-15\% reduction. SEER Cancer Statistics Review 1975-2007)

changes in the age-adjusted breast cancer mortality rate. ${ }^{1}$ The size tipping point inferred from the temporal relationship between the $2.5-\mathrm{cm}$ mean and $2.0-\mathrm{cm}$ median maximum diameters and the onset of mortality decline should be a stimulus not just to achieve these apparent size break points leading to the onset of mortality decline as noted, but also to reduce size still further to the median maximum diameter of about $1.0 \mathrm{~cm}$, which is achieved in all mammographically discovered invasive breast cancers with yearly screening, compared to $1.5 \mathrm{~cm}$ now recorded for entire populations. ${ }^{11}$

A unique demonstration of mortality decline with screening occurred in the 1970s in Rhode Island, one of the initial five sites of the Breast Cancer Detection Demonstration Project (BCDDP). ${ }^{12}$ The Rhode Island site was funded and began in 1974 and lasted for 5 years, the BCDDP funding duration. In 1978, 4 years after the Rhode Island BCDDP project started, breast cancer mortality began to decline, reaching a temporary nadir in 1981. After funding ended in 1978 and mammography was deemphasized nationally because of concerns regarding radiation and quality of equipment, the Rhode Island mortality rate returned to baseline by 1985 (Fig. 4). The impact of the 29 eventual nationwide sites of BCDDP was submerged in the large overall population, whereas in the small state of Rhode Island, the mortality impact, as noted in the cumulative BCDDP reports, was demonstrated temporally on a 

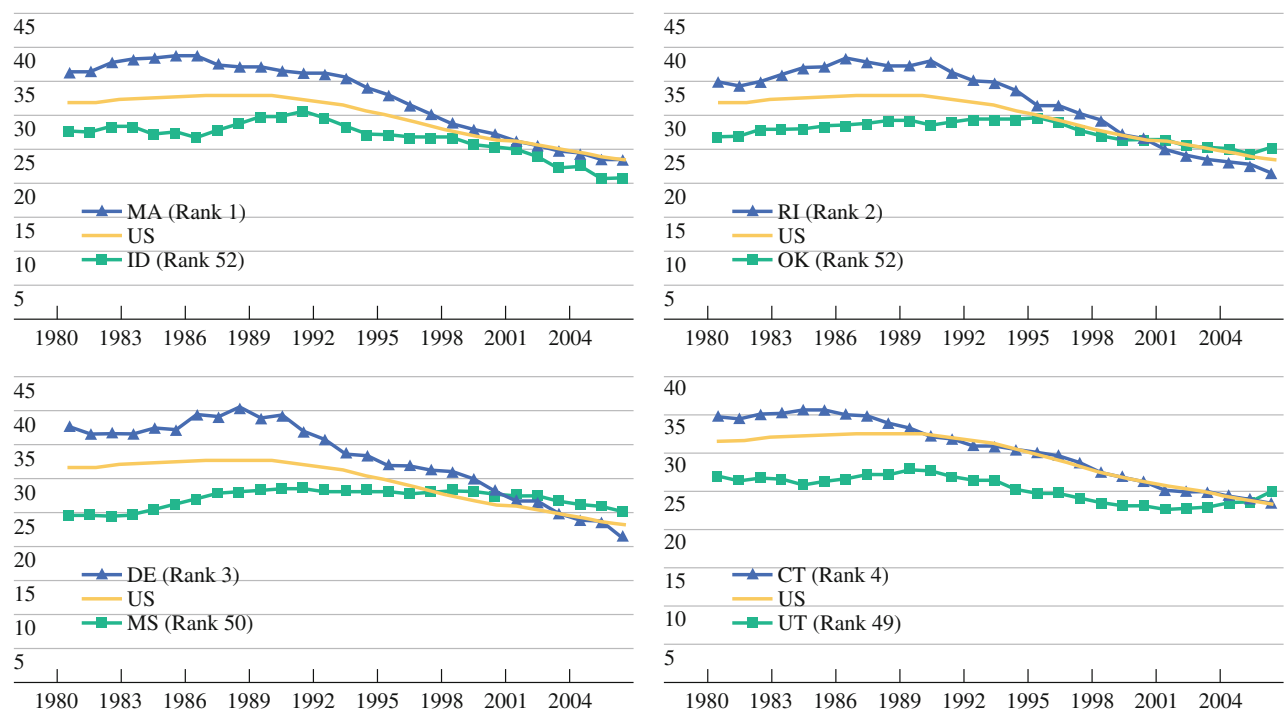

FIG. 3 Comparison of breast cancer mortality in states with high and low rates of mammographic screening. Note the more substantial mortality decline as ranked by the Behavioral Risk Factor Surveillance System (BRFSS) 2006 survey of state mammography use at least every 2 years in women over 40 , comparing the top four states $(\mathrm{MA}=84.8 \% ; \mathrm{RI}=84.5 \% ; \mathrm{DL}=83.7 \% ; \mathrm{CT}=82.0 \%)$ to the

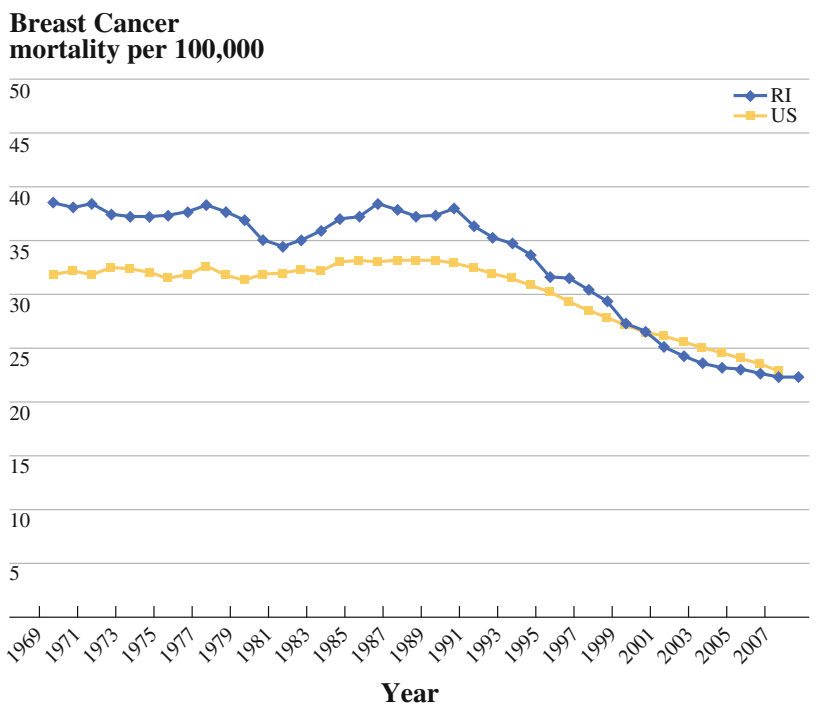

FIG. 4 Impact of screening mammography programs on breast cancer mortality. Age-standardized female breast cancer mortality rates for the State of Rhode Island were constructed by SEERStat software, available from the National Cancer Institute (http:// seer.cancer.gov/seerstat/), and based on preloaded death records from the National Center for Health Statistics. Breast cancer mortality is reported per 100,000 with running 5-year averages illustrated. Note the temporary mortality decrease in Rhode Island after 1977 until 1981 (38.2-34.3) after activation of the Rhode Island site (one of the first 5) of the Breast Cancer Detection Demonstration Project (BCDDP) in 1974. BCDDP funding ceased in Rhode Island in 1978 at expiration of the 5-year grant. Mortality then returned to baseline by 1986 (38.3) until 1990 (37.9) followed by the continuing decrease in mortality that began after 1990 until 2008 (22.3) for a total mortality decline of $41 \%$ (37.9-22.3)

bottom four states $(\mathrm{ID}=67.3 \% ; \quad \mathrm{OK}=67.7 \% ; \quad \mathrm{MS}=67.8 \%$; $\mathrm{UT}=68.7 \%$ ). Breast cancer mortality expressed deaths $/ 100,000 /$ year, and mammography screening expressed as proportion of women over 40 having a mammogram within 2 years (Graphs prepared by Jarurat Ousinasawat from data published on http://statecancerprofiles. cancer.gov)

statewide level. Subsequently, after the later upsurge in mammographic screening in the 1980s, breast cancer mortality in Rhode Island again began to decline, but then continued as mass screening continued. ${ }^{10}$

Taken together, the smaller size, decreased node metastatic rate, and fewer high-grade cancers provide evidence of an interruption in the progressive biological evolution of most breast cancer. ${ }^{9}$ The earlier presentation by population screening that created the tipping point of downward deflection of breast cancer mortality that is now continuing at a rate of almost a $2 \%$ decrease in age-adjusted mortality per year in the United States is nothing short of a dramatic public health achievement. ${ }^{1}$ This will probably enable the American Cancer Society challenge goal of a $50 \%$ decline in mortality by 2015 to be met. ${ }^{13}$ States and populations with high mammographic screening rates, as noted, will undoubtedly exceed that goal. All this is a testament to the power of the early detection by mammographic screening of breast cancer that has changed the course of this progressively evolving cancer in entire populations, states, and countries.

There is clear evidence, even in the United States Preventive Services Task Force (USPSTF) models recently published, that yearly screening is more effective than the biennial screening they recommended both in mortality reduction and life years gained. ${ }^{14}$ In contrast to the "best" biennial model promoted by the USPSTF, a further $70 \%$ proportional improvement in both mortality reduction and life years gained by screening was achieved in their model 
of annual age 40 to 84 mammographic screening compared to their recommended biennial screening from 50 to 74. ${ }^{14}$ Of great importance, yearly screening reduces the incidence, size, node metastatic rate, and mortality of cancers that appear as palpable masses between regularly screenings (interval cancers) compared to every-otheryear screenings, a factor not acknowledged by the USPSTF report but well established in actual patient data, statistical modeling, and evaluating deaths from breast cancer. $^{11,14-17}$ With screening every 2 years, the $25 \%$ of cancers that are interval cancers are similar to breast cancers diagnosed before mammography was used.,.$^{3,411}$ Just as "yearly" schedules actually occur at about 16-month median intervals, "biennial" screening will undoubtedly be closer to 30-month intervals as a result of patient forgetfulness, difficulty booking mammograms, and other factors. ${ }^{16,17}$

Why did the USPSTF deliberately choose a less effective screening schedule? Their conclusion implied that resource conservation was more important than maximum mortality reduction in their calculations. They failed to point out that the more intense screening-25,000 more mammograms per 1000 women when comparing annual (40-84 years) to biennial (50-74 years) screening-might well be cost neutral when balanced against the extra lives saved and life-years gained. Twenty-five thousand extra mammograms per 1000 women at \$80 each (\$2 million) might well be offset, in whole or in large part, by the estimated $\$ 50,000$ to $\$ 100,000$ per life saved (\$1.1 million to $\$ 2.2$ million), calculating that 22 extra lives would be saved per 1000 women by the $70 \%$ proportional increased mortality reduction. Why the USPSTF deliberately chose a less effective method of preventing mortality in the most frequent and most feared cancer of women is a puzzle, especially as cost considerations may not be a major adverse factor, although resource allocation is increased.

In conclusion, yearly mammographic screening, which has been demonstrated to further reduce mortality by detecting smaller breast cancers and by reducing the incidence of more common and more lethal interval cancers that occur in every other year (biennial) screening, should be the standard recommendation for women over 40 . The eventual reduction of breast cancer mortality to less than $5 \%$ can be achieved today in women who are regularly screened at yearly intervals. ${ }^{17}$ This approximate $5 \%$ mortality rate was also achieved in the 1960s among cancers discovered in the BCDDP screening initiative. ${ }^{12}$

ACKNOWLEDGMENT The authors acknowledge Jarurat Ousinasawat for her assistance in creating Fig. 3.

\section{REFERENCES}

1. Jemal A, Siegel R, Xu J, Ward E. Cancer Statistics, 2010. CA: A Cancer J Clin. 2010. doi:10.3322/caac.20073.

2. Torio CM, Klassen AC, Curriero FC, Caballero B, Helzlsouer K. The modifying effect of social class on the relationship between body mass index and breast cancer incidence. Am J Public Health. 2010;100:146-51

3. Cady B, Stone MD, Schuler JG, Thakur R, Wanner MA, Lavin PT. The new era in breast cancer. Invasion, size, and nodal involvement dramatically decreasing as a result of mammographic screening. Arch Surg. 1996;131:301-8.

4. Coburn NG, Chung MA, Fulton J, Cady B. Decreased breast cancer tumor size, stage, and mortality in Rhode Island: an example of a well-screened population. Cancer Control. 2004;11: 222-30.

5. Coburn NG, Fulton JP, Cady B, Law C, Chung MA. Continuously improving size, lymph node metastatic rate, and mortality of invasive breast cancer in Rhode Island women, a well screened population. Cancer. Submitted.

6. Gur AS, Unal B, Johnson R, et al. Predictive probability of four different breast cancer nomograms for nonsentinel axillary lymph node metastasis in positive sentinel node biopsy. J Am Coll Surg. 2009;208:229-35.

7. Giuliano AEML, Beitsch P, Whitworth PW, et al. Local and regional control in breast cancer after sentinel node biopsy without axillary node dissection: results from a randomized trial. Ann Surg. 2010;252:426-32.

8. Feinstein AR, Sosin DM, Wells CK. The Will Rogers phenomenon. Stage migration and new diagnostic techniques as a source of misleading statistics for survival in cancer. $N$ Engl J Med. 1985;312:1604-8.

9. Hellman S. Karnofsky Memorial Lecture. Natural history of small breast cancers. J Clin Oncol. 1994;12:2229-34.

10. Gaudette LA, Altmayer CA, Nobrega KM, Lee J. Trends in mammography utilization, 1981 to 1994. Health Rep. 1996;8: $17-27$.

11. Hunt KA, Rosen EL, Sickles EA. Outcome analysis for women undergoing annual versus biennial screening mammography: a review of 24,211 examinations. AJR Am J Roentgenol. 1999;173: 285-9.

12. Smart CR, Byrne C, Smith RA, et al. Twenty-year follow-up of the breast cancers diagnosed during the Breast Cancer Detection Demonstration Project. CA Cancer J Clin. 1997;47:134-49.

13. Byers T, Mouchawar J, Marks J, et al. The American Cancer Society challenge goals. How far can cancer rates decline in the U.S. by the year 2015? Cancer. 1999;86:715-27.

14. Nelson HD, Tyne K, Naik A, Bougatsos C, Chan BK, Humphrey L. Screening for breast cancer: an update for the U.S. Preventive Services Task Force. Ann Intern Med. 2009;151:727-37.

15. Michaelson JS, Satija S, Kopans D, et al. Gauging the impact of breast carcinoma screening in terms of tumor size and death rate. Cancer. 2003;98:2114-24.

16. Spencer DB, Potter JE, Chung MA, Fulton J, Hebert W, Cady B. Mammographic screening and disease presentation of breast cancer patients who die of disease. Breast J. 2004;10:298-303.

17. Cady B, Webb M, Michaelson JS, Kopans D, Smith BL. Women who die of breast cancer are predominantly those not participating in regular mammographic screening. Presented at American Society of Clinical Oncology Breast Cancer Meeting, September. 2009, San Francisco, CA. 